\title{
Isaac Newton \& Émilie du Châtelet, Principes mathématiques de la philosophie naturelle. La traduction française des Philosophiae naturalis principia mathematica
}

\section{Debora Sicco}

\section{(2) OpenEdition}

\section{Journals}

Edizione digitale

URL: http://journals.openedition.org/studifrancesi/9957

DOI: 10.4000/studifrancesi.9957

ISSN: 2421-5856

Editore

Rosenberg \& Sellier

\section{Edizione cartacea}

Data di pubblicazione: 1 août 2017

Paginazione: 360

ISSN: 0039-2944

\section{Notizia bibliografica digitale}

Debora Sicco, «Isaac Newton \& Émilie du Châtelet, Principes mathématiques de la philosophie naturelle. La traduction française des Philosophiae naturalis principia mathematica», Studi Francesi [Online], 182 (LXI | II) | 2017, online dal 01 août 2017, consultato il 06 janvier 2021. URL: http://journals.openedition.org/ studifrancesi/9957 ; DOI: https://doi.org/10.4000/studifrancesi.9957

Questo documento è stato generato automaticamente il 6 janvier 2021 .

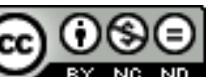

Studi Francesi è distribuita con Licenza Creative Commons Attribuzione - Non commerciale - Non opere derivate 4.0 Internazionale. 


\title{
Isaac Newton \& Émilie du Châtelet, Principes mathématiques de la philosophie naturelle. La traduction française des Philosophiae naturalis principia mathematica
}

\author{
Debora Sicco
}

\section{NOTIZIA}

ISAAC NEWTON \& ÉMILIE du CHÂTELET, Principes mathématiques de la philosophie naturelle. La traduction française des Philosophiae naturalis principia mathematica, a cura di Michel Toulmonde, Ferney-Voltaire, Centre international d'étude du XVIII ${ }^{\mathrm{e}}$ siècle, 2015, 2 voll., $524+556 \mathrm{pp}$.

1 In questi due eleganti volumi, Michel Toulmonde presenta l'edizione critica del manoscritto autografo della traduzione francese dei Philosophiae naturalis principia mathematica di Newton realizzata da Gabrielle-Émilie Le Tonnelier de Breteuil, marchesa Du Châtelet (1706-1749). Si tratta di un progetto editoriale nato in occasione del convegno organizzato presso la Bibliothèque Nationale de France nel giugno 2006, per il tricentenario della nascita della marchesa. Oltre ai due volumi qui presentati, ne è previsto un terzo, dedicato al Commentaire dell'opera newtoniana redatto da Émilie insieme alla traduzione.

2 Nei Principia, scritti su sollecitazione di Halley per approfondire le questioni affrontate nel De motu, Newton ha introdotto l'idea di gravitazione universale e ha criticato la teoria cartesiana dei tourbillons. Nonostante l'immediato successo ottenuto in Inghilterra, dovranno passare circa 70 anni dalla prima edizione dei Principia perché le idee innovative di Newton siano definitivamente accettate dall'Europa colta. 
All'edizione del 1687 segue una seconda nel 1713, con modifiche e integrazioni suggerite dal fisico Roger Cotes, e una terza nel 1726. Émilie ha lavorato su quest'ultima edizione dei Principia, frutto della collaborazione fra Newton e Henry Pemberton. Il progetto di tradurre dal latino i Principia e commentarli ha preso forma nel 1745: dopo mesi di intenso lavoro, il manoscritto della traduzione è inviato alla Censura, dove riceve presto l'approvazione di Clairaut. All'inizio del 1749, poco prima di partorire, Madame du Châtelet farà depositare questo manoscritto alla Biblioteca del re. Oltre ad esso, i manoscritti dei due volumi pubblicati nel 1759 comprendono un Commentaire e le figure per i libri II e III. Gli originali di queste ultime (preparati e numerati da Émilie) sono stati ritrovati con gli archivi della famiglia du Châtelet soltanto nel 2010, insieme alla prima parte del Commentaire.

3 Sulla base di questi documenti, Michel Toulmonde ha condotto un accurato lavoro, corredato da un'introduzione in cui descrive la struttura dei manoscritti e si sofferma sui vari elementi utili a una migliore comprensione del testo, come l'ortografia, i mutamenti intercorsi nelle espressioni matematiche dal xvIII secolo a oggi, gli errori presenti nelle circa 1400 pagine del manoscritto, la scrittura di Madame du Châtelet, le figure e le tabelle con i valori numerici. Il curatore prova infine a spiegare le ragioni della tardiva pubblicazione dei Principes mathématiques, usciti soltanto il 21 giugno 1759; a questo ritardo non sarebbero estranei, accanto a notevoli difficoltà tipografiche relative alle planches, gli impegni di Clairaut - principale responsabile della pubblicazione del Commentaire - e l'assenza dalla Francia di Voltaire, compagno di Émilie dal 1733 e come lei impegnato nella diffusione delle idee di Newton. Fra coloro che vi hanno contribuito - oltre a Clairaut, si ricordano anche Maupertuis e Algarotti Émilie ha senza dubbio rivestito un ruolo di primo piano e questa traduzione, ora disponibile in un'edizione critica moderna, lo dimostra. 